\title{
The Central American tree snake, Imantodes gemmistratus (Cope, 1861) (Squamata: Dipsadinae): a new record for Zacatecas, Mexico
}

\author{
Jorge A. Bañuelos-Alamillo", ${ }^{1,}$, Ilse Yasareth Trujillo-De la Torre ${ }^{2}$, Gustavo E. Quintero-Díaz ${ }^{3,5}$ \& \\ Rubén Alonso Carbajal-Márquez ${ }^{4,5,6}$ \\ ${ }^{1}$ Unidad Académica de Ciencias Biológicas, Universidad Autónoma de Zacatecas, Edificio de Biología Campus II Ave. Preparatoria S/N, \\ Col. Agronómica, 98066, Zacatecas, Zacatecas, Mexico \\ ${ }^{2}$ Centro de Bachillerato Tecnológico Agropecuario No. 167 “Gral. J. Jesús González Ortega”, Laboratorio de Biología, Km.1 Camino a \\ Agua Fría, Col. Agua Fría, Valparaíso, Zacatecas, Mexico \\ ${ }^{3}$ Universidad Autónoma de Aguascalientes, Centro de Ciencias Básicas, Departamento de Biología. C. P. 20131, Aguascalientes, \\ Aguascalientes, Mexico \\ ${ }^{4}$ El Colegio de la Frontera Sur. Departamento de Conservación de la Biodiversidad. Unidad Chetumal, Av. Centenario Km 5.5, 77014, \\ Chetumal, Quintana Roo, Mexico \\ ${ }^{5}$ Conservación de la Biodiversidad del Centro de México, A. C. Andador Torre de Marfil No. 100, C. P. 20229, Aguascalientes, \\ Aguascalientes, Mexico \\ ${ }^{6}$ Corresponding author. E-mail: redman031@hotmail.com
}

\begin{abstract}
We document the first record of Imantodes gemmistratus for Zacatecas state, Mexico. In August 2016, one adult male was found in a dry forest and oak forest ecotone in the Municipality of Valparaiso. This new record extends known distribution of the species $166 \mathrm{~km}$ to the northeast from the nearest previous record in Nayarit state.
\end{abstract}

Key words. Sierra Madre Occidental; dry forest; oak forest; first record; reptile; arboreal snake

The snake genus Imantodes is distinguished by a very slender body, a large head clearly distinct from the neck, and large protruding eyes. These snakes are crepuscular and nocturnal shrub dwellers, and during night they can be seen usually stretched out, gliding slowly through the vegetation. Anole lizards (Anolis) are their main prey, but they also consume small frogs and eggs. All the species of this genus are oviparous (KöHLER 2008). The genus comprises 8 species (UETZ et al. 2016), among them Imantodes gemmistratus (Cope, 1861), which presents a wide distributional range in northern and central America, from southwestern Chihuahua and southern Sonora (Mexico), along the Pacific versant to Colombia, and from northern Veracruz through the Atlantic versant to Quintana Roo, with an isolated record in the Atlantic versant of Costa Rica (Zweifel 1959, García \& Ceballos 1994, KöHLer 2008, Lemos-EsPinal \& SMith 2009, RamíreZBAutista et al. 2014). In Mexico, this species inhabits tropical dry, moist, and coniferous forests of Campeche, Chiapas, Chihuahua, Colima, Estado de México, Guerrero, Hidalgo, Jalisco, Michoacán, Morelos, Nayarit, Oaxaca, Puebla, Quintana Roo, Sinaloa, Sonora, Tabasco, Veracruz, and Yucatán states, from sea level to $2,000 \mathrm{~m}$ above sea level (ZweIFEL
1959, Álvarez del Toro 1982, Myers 1982, García \& CebalLos 1994, Ramírez-Bautista 1994, Lemos-EsPinal \& Smith 2009, CANSECo-MÁrquez \& GuTIÉRREZ-MAYÉN 2010). Imantodes gemmistratus is listed in Mexico as "Sujeta a Protección Especial" (subject to special protection) by the government's list of threatened species (Diario Oficial de La Federación 2010), but it has not been evaluated by the IUCN. Here, we report the first record of the Central American Tree Snake, I. gemmistratus from Zacatecas state, Mexico.

An adult male I. gemmistratus (Fig. 1) was found alive on 4 August 2016 at $23: 00 \mathrm{~h}, 19.5 \mathrm{~km}$ southwest of Valparaiso, Municipality of Valparaiso, Zacatecas $\left(22.6847^{\circ} \mathrm{N}\right.$, $103.7345^{\circ} \mathrm{W}$ [WGS84]; $2,270 \mathrm{~m}$ above sea level). The adult male was collected on a road surrounded by dry forest-oak forest ecotone. This vegetation is composed of Huizache (Acacia farnesiana), Fern-leaf Acacia (A. pennatula), Catclaw Mimosa (Mimosa monancistra), Palo Blanco (Ipomoea intrapilosa), Cazahuate (I. murucoides), Prickly Pear (Opuntia fuliginosa), oaks (Quercus spp.) and Pointleaf Manzanita (Arctostaphylos pungens) (Sousa \& MarTínez 2010). The specimen identification was verified by Bradford Hollingsworth and a photo was deposited in the San Diego Natural History Museum (SDSNH_HerpPC_05340). The specimen itself was collected under the permit SEMARNAT SGPA/DGVS/03079/16 and deposited in the Vertebrate Collection of the Universidad Autónoma de Aguascalientes (CZ-UAA-REP-0645). Locality records for I. gemmistratus were obtained from the Global Biodiversity Information Facility and literature (GBIF 2016; Appendix Table A1).

Imantodes gemmistratus is a slender snake reaching 880 $\mathrm{mm}$ in total length (TL). The ventral scales in the populations from northwestern Mexico number 220-237, the subcaudals 


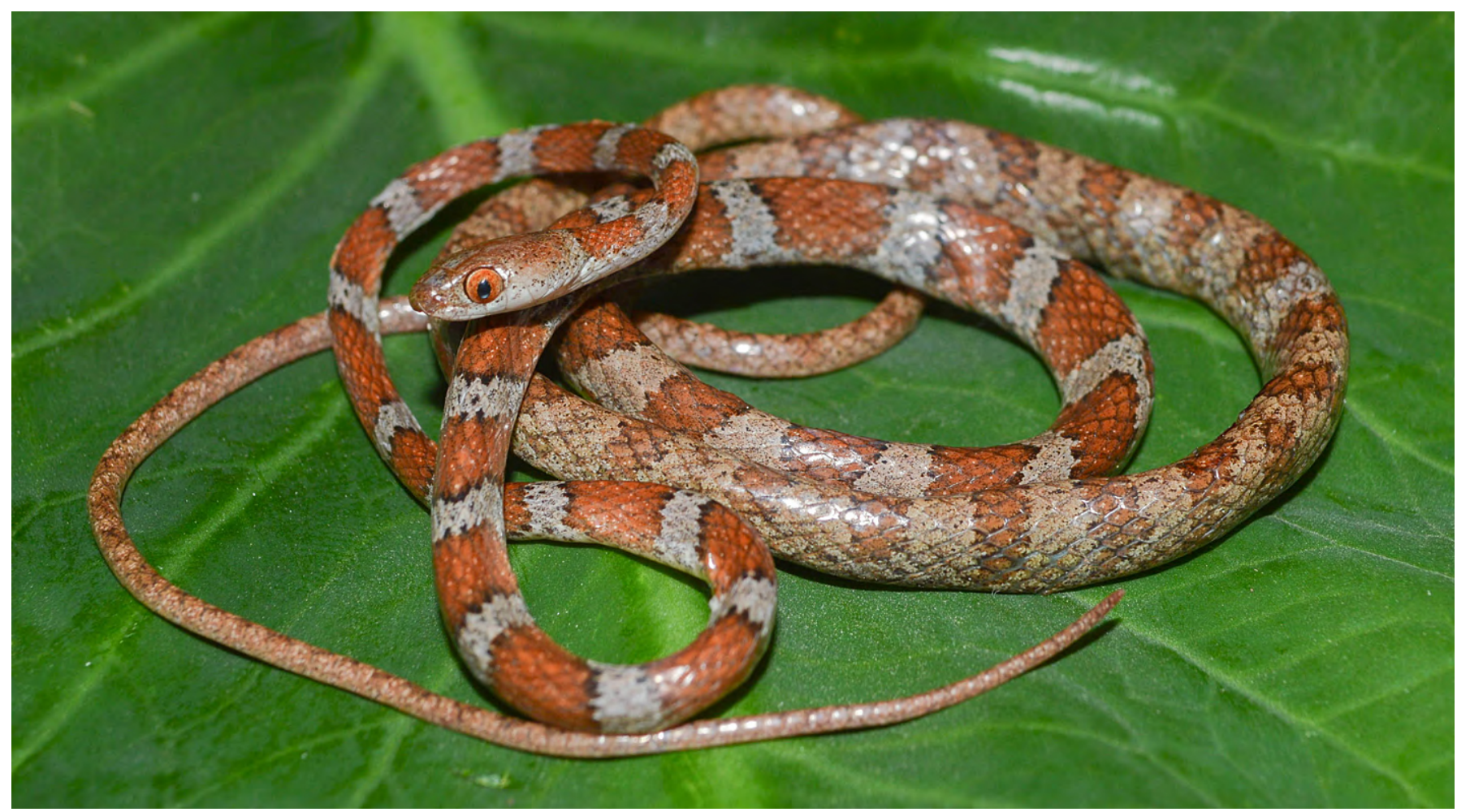

Figure 1. Adult male specimen CZ-UAA-REP-0645 of Imantodes gemmistratus from Valparaiso, Zacatecas, Mexico (SDSNH_HerpPC_05340).

114-130 in males and 109-129 in females (Lemos-EsPinAL \& SмITH 2009). The head scales are normal, with 1 loreal and 1 preocular, a singular anterior temporal, usually 8 supralabials (4, 5 and 6 entering the orbit) and 9 infralabials. The internasals are about $1 / 3$ the size of the prefrontals. Individuals of this species possess large and protruding eyes whose diameter is 3 times their distance from the lip. The snake has grooved rear fangs and a vertical pupil. The head is pale tan without markings. There are 36-53 large, light reddish brown dark edged blotches on the body, narrowing on the sides, all reaching the edges of the ventrals, but becoming lighter posteriorly so that they cannot be discerned. The interspaces are light gray or brown. The tail is more or less uniformly brownish above (Zweifel 1959, Savage \& Scott 1985; Lemos-Espinal $\&$ SMith 2009). Imantodes gemmistratus is the only arboreal snake in the state with such a slim body and a noticeable difference between the neck and head with a blunt snout and large eyes. The male specimen from Zacatecas (snout-vent length $=455 \mathrm{~mm}, \mathrm{TL}=655 \mathrm{~mm}$; Fig. 1) presents 8 supralabials (4, 5 and 6 entering the orbit) and 8 infralabials, 210 ventrals, 119 subcaudals, an undivided anal plate, and 52 dorsal body blotches. All characters coincide with the available descriptions of Mexican individuals except the lower number of ventral scales. However, still lower ventral counts have been obtained across the species' entire range (KöHLER 2008).

This is the first record of this species for Zacatecas state and extends its known distribution by $166 \mathrm{~km}$ northeast in a straight-line from " $4.1 \mathrm{~km}$ northwest [of] Rosamorada, Nayarit” (LACM-103516; GBIF 2016; Fig. 2). The nominal species I. gemmistratus is likely a species complex with many lineages currently recognized as 7 subspecies (ZWEIFEL 1959). Our specimen corresponds to the northwestern form (I. $g$.
latistratus[Cope 1887]). This new record and those records of other reptile species previously recorded (BAÑUELOSAlamillo et al. 2015a, b, Carbajal-Márquez et al. 2015, Bañuelos-Alamillo \& CARbajal-Márquez 2016, BañueLOS-AlAmillo et al. 2016) suggest that additional species typical of the Pacific slope likely remain to be documented in Zacatecas. It also reinforces the importance of conserving the canyons of the upper Río Grande de Santiago basin that serve as biological corridors.

\section{LITERATURE CITED}

Álvarez del Toro, M. 1982. Los reptiles de Chiapas. Colección Libros de Chiapas. Tuxtla Gutiérrez: Publicación del Instituto de Historia Natural. 248 pp.

Bañuelos-Alamillo, J.A., Carbajal-Márquez, R.A., QuinteroDíAz, G.E. \& OchOA, G.M. 2015a. Heloderma horridum (Mexican Beaded Lizard): geographic distribution. Herpetological Review 46: 383.

Bañuelos-Alamillo, J.A., Carbajal-Márquez, R.A., QuinteroDíAz, G.E. \& OCHOA, G.M. 2015b. Trimorphodon paucimaculatus (Sinaloan Lyre Snake): geographic distribution. Herpetological Review 46: 387.

Bañuelos-Alamillo, J.A. \& Carbajal-Márquez, R.A. 2016. Hypsiglena affinis: geographic distribution. Mesoamerican Herpetology 3: 198.

Bañuelos-Alamillo, J.A., Carbajal-Márquez, R.A. \& RojoCarrillo, G. 2016. Boa imperator: geographic distribution. Mesoamerican Herpetology 3: 184.

Canseco-Márquez, L. \& Gutiérrez-Mayén, M.G. 2010. Anfibios y reptiles del Valle de Tehuacán-Cuicatlán. México, DF: Comisión Nacional para el Conocimiento y Uso de la Biodiversidad, Fundación para la Reserva de la Biosfera Cuicatlán A.C., Benemérita Universidad Autónoma de Puebla. 302 pp. 


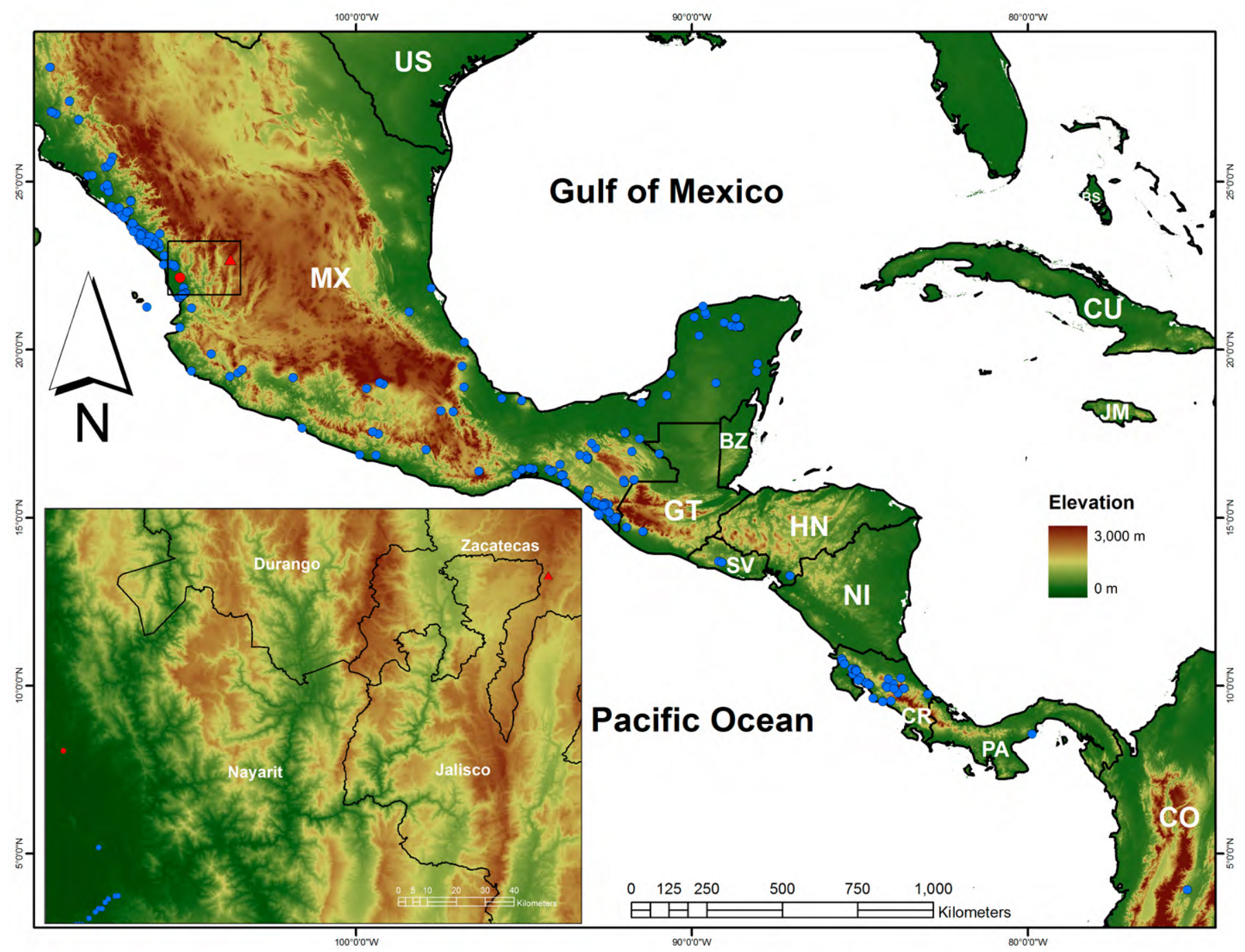

Figure 2. Collecting localities for Imantodes gemmistratus across its geographic range. Blue dots show previous records (see Appendix Table A1). The red triangle represents our new record of I. gemmistratus for Zacatecas at Valparaiso, the red circle the nearest previously reported locality at Nayarit. Country codes are as follows: $\mathrm{BS}=$ Bahamas, $\mathrm{BE}=$ Belize, $\mathrm{CO}=$ Colombia, $\mathrm{CR}=$ Costa Rica, $\mathrm{CU}=\mathrm{Cuba}$, GT = Guatemala, $\mathrm{HN}=\mathrm{Honduras}, \mathrm{JM}=\mathrm{Jamaica}$, $\mathrm{MX}=$ Mexico, $\mathrm{NI}=$ Nicaragua $\mathrm{PA}=$ Panama, $\mathrm{SA}=$ El Salvador, $\mathrm{US}=$ United States.

Carbajal-Márquez, R.A., Bañuelos-Alamillo, J.A., RivasMercado, E.A., Quintero-Díaz, G.E. \& Domínguez de la Riva, M.A. 2015. Crotalus basiliscus (Mexican West Coast Rattlesnake): geographic distribution. Herpetological Review 46: 385.

Cope, E.D. 1861. Contributions to the ophiology of Lower California, Mexico and Central America. Proceedings of the Academy of Natural Sciences of Philadelphia 13: 292-306.

Diario Oficial de la Federación. 2010. Norma Oficial Mexicana NOM-059-SEMARNAT-2010, Protección ambiental-Especies nativas de México de flora y fauna silvestres-Categorías de riesgo y especificaciones para su inclusión, exclusión o cambioLista de especies en riesgo, 30 de diciembre de 2010. Accessed at http://www.profepa.gob.mx/innovaportal/file/435/1/NOM_059 SEMARNAT_2010.pdf, 21 February 2017.

García, A. \& Ceballos. G. 1994. Guía de campo de los reptiles y anfibios de la costa de Jalisco, México. México, DF: Fundación Ecológica de Cuixmala A. C., Instituto de Biología UNAM. 184 pp.

GBIF (Global Biodiversity Information Facility). 2016. GBIF Occurrence download. Accessed at https://oi.org/10.15468/ dl.dkdgzz

KöHLER, G. 2008. Reptiles of Central America. 2nd edition. Offen- bach: Herpeton Verlag. 400 pp.

Lemos-Espinal, J.A. \& Sмith, H.M. 2009. Amphibians and reptiles of the state of Chihuahua, Mexico. México, DF: UNAM, CONABIO, University of Colorado at Boulder. 613 pp.

Myers, C.W. 1982. Blunt-headed vine snakes (Imantodes) in Panama, including a new species and other revisionary notes. American Museum Novitates 2738: 1-50.

Ramírez-Bautista, A.1994. Manual y claves ilustradas de los anfibios y reptiles de la región de Chamela, Jalisco, México. Cuadernos del Instituto de Biología 23. México, DF: Universidad Nacional Autónoma de México. 127 pp.

Ramírez-Bautista, A., Hernández-Salinas, U., Cruz-Elizalde, R., Berriozabal-Islas C., Lara-Tufiño, D., Goyenechea, I. \& Castillo-Cerón, J. 2014. Los anfibios y reptiles de Hidalgo, México: diversidad, biogeografía y conservación. Pachuca: Universidad Autónoma del Estado de Hidalgo y Sociedad Herpetológica Mexicana. 387 pp.

Savage, J.M. \& ScotT, N.J. 1985. The Imantodes (Serpentes: Colubridae) of Costa Rica: two or three species? Revista de Biología Tropical 33: 107-132.

Sousa, M. \& Martínez, E. 2010. Cuenca Alta del Río Santiago, Jalisco, Zacatecas, Nayarit y Durango; pp. 421-423, in: CebAllos, G., Martínez, L., García, A., Espinoza, E., Bezaury-Creel, 
J. \& DiRzo, R. (eds.). Diversidad, amenazas, y áreas prioritarias para la conservación de las selvas secas del Pacífico de México. México, DF: Fondo de Cultura Económica, Comisión Nacional para el Conocimiento y Uso de la Biodiversidad.

Uetz, P., Freed, P. \& Hošek, J. 2016. The reptile database. Accessed at http://www.reptile-database.org, 21 February 2017.

Zweifel, R.G. 1959. Snakes of the genus Imantodes in western Mexico. American Museum Novitates 1961: 1-17.
Authors' contributions. JABA, RACM, GEQD and IYTD collected the data. RACM, GEQD and JABA wrote the text. GEQD made the map and JABA took the photographs.

Received: 22 September 2016

Accepted: 5 April 2017

Academic editor: Sebastian Lotzkat

\section{APPENDIX}

Table A1. Records of Imantodes gemmistratus obtained from the present study, published literature and museum specimens consulted in GBIF. AMNH: American Museum of Natural History; CAS: California Academy of Science; CM: Carnegie Museum of Natural History; CUMV: Cornell University Museum of Vertebrates; ECOSUR-CH: El Colegio de la Frontera Sur-Chetumal; IHN: Instituto de Historia Natural-Chiapas; KU: University of Kansas Natural History Museum; LACM: Natural History Museum of Los Angeles County; MCZ: Museum of Comparative Zoology; MNHUK: Museum of Natural History University of Kansas; MLS: Museo de La Salle; MZFC: Museo de Zoología Facultad de Ciencias-UNAM; SBMNH: Santa Barbara Museum of Natural History; SDNHM: San Diego Natural History Museum; TCWC: Texas Cooperative Wildlife Collection; UCM: University of Colorado Museum; UMMZ: University of Michigan Museum of Zoology; USNM: National Museum of Natural History, Smithsonian Institution; UTEP: University of Texas at El Paso. Country codes are $\mathrm{BS}=$ Bahamas, $\mathrm{BE}=$ Belize, $\mathrm{CO}=$ Colombia, $\mathrm{CR}=$ Costa Rica, $\mathrm{CU}=\mathrm{Cuba}, \mathrm{GT}=$ Guatemala, $\mathrm{HN}=$ Honduras, $\mathrm{JM}=$ Jamaica, $\mathrm{MX}=\mathrm{Mexico}, \mathrm{NI}=\mathrm{Nicaragua}$ $\mathrm{PA}=$ Panama, $\mathrm{SA}=$ El Salvador, US = United States.

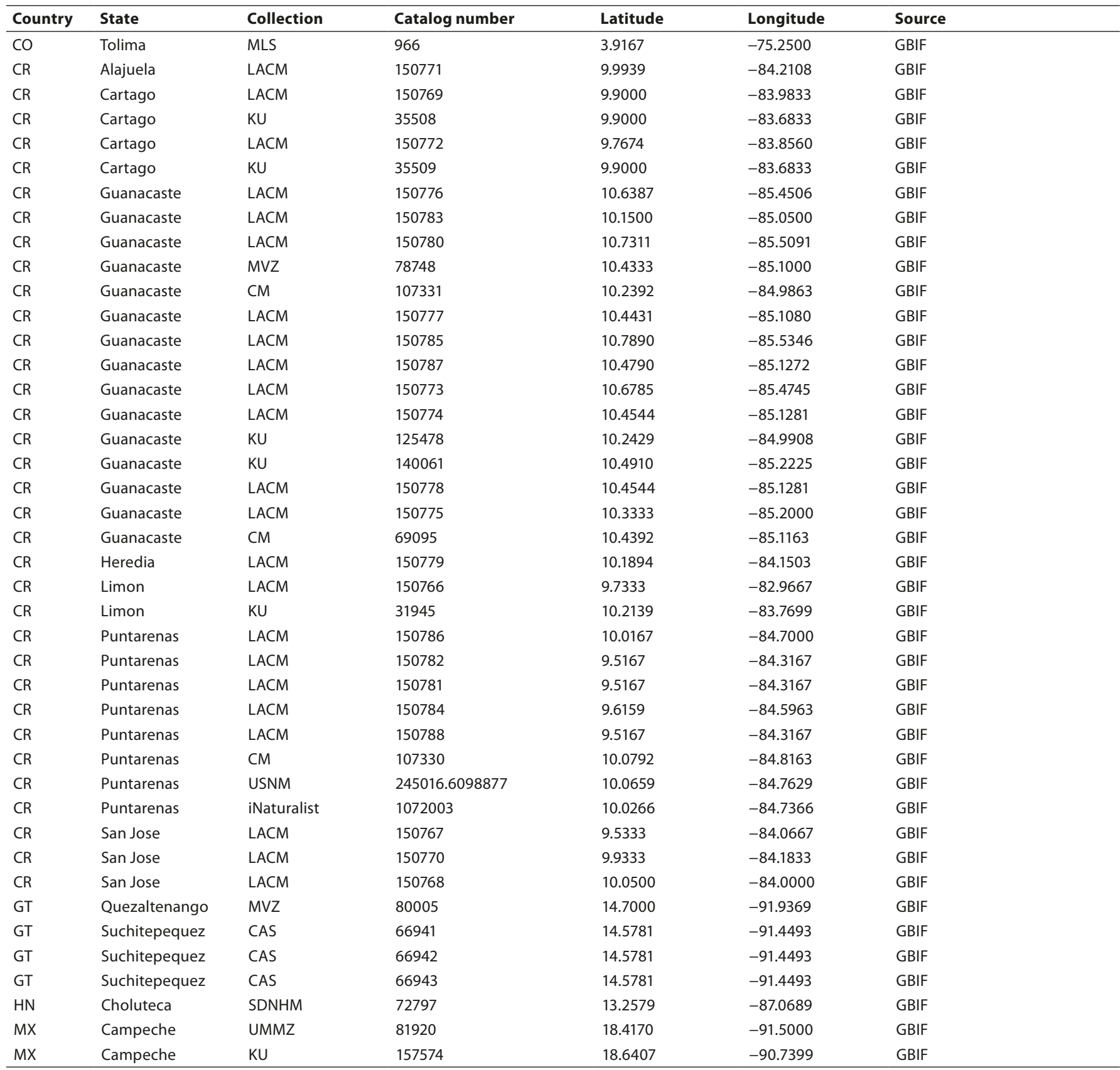


Table 1. Continued.

\begin{tabular}{|c|c|c|c|c|c|c|}
\hline Country & State & Collection & Catalog number & Latitude & Longitude & Source \\
\hline$M X$ & Campeche & UMMZ & 81921 & 18.4170 & -91.5000 & GBIF \\
\hline MX & Campeche & ECOSUR-CH & 960 & 19.0020 & -89.2800 & GBIF \\
\hline MX & Campeche & IHN & 1124 / 4348 / V050 & 19.2720 & -90.6230 & GBIF \\
\hline MX & Chiapas & IHN & 662 / 5518 / V050 & 15.0990 & -92.7870 & GBIF \\
\hline$M X$ & Chiapas & MVZ & 177761 & 15.1500 & -92.4600 & GBIF \\
\hline MX & Chiapas & USNM & 110524 & 17.5080 & -91.9810 & GBIF \\
\hline MX & Chiapas & UTEP & $\mathrm{H}-9519$ & 16.2508 & -93.8800 & GBIF \\
\hline$M X$ & Chiapas & MVZ & 112407 & 15.0078 & -92.2485 & GBIF \\
\hline MX & Chiapas & MVZ & 177761 & 15.1508 & -92.4452 & GBIF \\
\hline MX & Chiapas & LACM & 38189 & 15.6180 & -93.1070 & GBIF \\
\hline MX & Chiapas & IHN & 737 / 264478 / U014 & 16.7580 & -93.1100 & GBIF \\
\hline MX & Chiapas & USNM & 110524.6082444 & 17.5424 & -91.9916 & GBIF \\
\hline MX & Chiapas & USNM & 110527.6082447 & 17.5424 & -91.9916 & GBIF \\
\hline MX & Chiapas & IHN & 74 / 4656 / V050 & 16.7530 & -93.1170 & GBIF \\
\hline MX & Chiapas & LSUMZ & 110524 & 17.5170 & -91.9830 & GBIF \\
\hline MX & Chiapas & LACM & 40549 / 263225 / U014 & 14.9350 & -92.2640 & GBIF \\
\hline MX & Chiapas & IUMNH & 33651 & 15.3620 & -92.6540 & GBIF \\
\hline MX & Chiapas & UC & 39696 & 15.3710 & -92.6000 & GBIF \\
\hline MX & Chiapas & USNM & 110526 & 17.5080 & -91.9810 & GBIF \\
\hline MX & Chiapas & LACM & 9503 & 15.4108 & -92.8299 & GBIF \\
\hline MX & Chiapas & USNM & 110523.6082443 & 17.5424 & -91.9916 & GBIF \\
\hline MX & Chiapas & LACM & 114140 & 15.2080 & -92.5640 & GBIF \\
\hline MX & Chiapas & IUMNH & 6286 & 16.0450 & -92.0200 & GBIF \\
\hline MX & Chiapas & IUMNH & 39147 & 16.5780 & -93.9250 & GBIF \\
\hline MX & Chiapas & LACM & 9503 / 265004 / U014 & 15.4610 & -92.9060 & GBIF \\
\hline MX & Chiapas & ND & 1847 / 266066 / U014 & 15.8020 & -93.0690 & GBIF \\
\hline MX & Chiapas & TCWC & 17168 & 16.1140 & -92.0250 & GBIF \\
\hline MX & Chiapas & USNM & 110525 & 17.5080 & -91.9810 & GBIF \\
\hline MX & Chiapas & ENCB & 12949 / 7283 / CC002 & 16.9010 & -90.9690 & GBIF \\
\hline MX & Chiapas & LSUMZ & 110527 & 17.5170 & -91.9830 & GBIF \\
\hline MX & Chiapas & $\mathrm{IHN}$ & 1599 & 15.0540 & -92.7450 & GBIF \\
\hline MX & Chiapas & CAS & 163730 & 16.2650 & -93.8269 & GBIF \\
\hline MX & Chiapas & UCM & 39696 & 17.2107 & -92.9800 & GBIF \\
\hline MX & Chiapas & $\mathrm{IHN}$ & 2644 & 15.4000 & -92.5800 & GBIF \\
\hline MX & Chiapas & USNM & 110521 & 15.3620 & -92.6210 & GBIF \\
\hline MX & Chiapas & IHN & 1456 / 13185 / V050 & 15.8020 & -93.0690 & GBIF \\
\hline MX & Chiapas & LSUMZ & 110523 & 17.5170 & -91.9830 & GBIF \\
\hline MX & Chiapas & USNM & 110525.6082445 & 17.5424 & -91.9916 & GBIF \\
\hline MX & Chiapas & MVZ & 132938 & 15.0020 & -92.2474 & GBIF \\
\hline MX & Chiapas & ENCB & 11142 & 16.8360 & -93.1440 & GBIF \\
\hline MX & Chiapas & UTEP & 9519 / 265586 / U014 & 16.2600 & -93.8760 & GBIF \\
\hline MX & Chiapas & MVZ & 112407 & 14.9120 & -92.3390 & GBIF \\
\hline$M X$ & Chiapas & USNM & 110521.6082441 & 29.6702 & -106.0903 & GBIF \\
\hline$M X$ & Chiapas & IHN & 654 / 264446 / U014 & 16.7580 & -93.1100 & GBIF \\
\hline $\mathrm{MX}$ & Chiapas & LACM & 9501 & 15.0009 & -92.3999 & GBIF \\
\hline$M X$ & Chiapas & $\mathrm{IHN}$ & 897 / 264646 / U014 & 16.7580 & -93.1100 & GBIF \\
\hline$M X$ & Chiapas & USNM & 110523 & 17.5080 & -91.9810 & GBIF \\
\hline $\mathrm{MX}$ & Chiapas & LACM & 38189 & 15.5708 & -93.1199 & GBIF \\
\hline$M X$ & Chiapas & $\mathrm{IHN}$ & 152 / 4816 / V050 & 16.7530 & -93.1170 & GBIF \\
\hline MX & Chiapas & $\mathrm{IHN}$ & 211 / 4927 / V050 & 16.7580 & -93.1100 & GBIF \\
\hline$M X$ & Chiapas & USNM & 110527 & 17.5080 & -91.9810 & GBIF \\
\hline$M X$ & Chiapas & $\mathrm{IHN}$ & 482 / 5287 / V050 & 15.3200 & -92.6610 & GBIF \\
\hline MX & Chiapas & $\mathrm{IHN}$ & 1108 / 264781 / U014 & 16.7890 & -93.0920 & GBIF \\
\hline $\mathrm{MX}$ & Chiapas & CAS & 163750 & 16.2789 & -93.8731 & GBIF \\
\hline$M X$ & Chiapas & LSUMZ & 110522 & 17.5170 & -91.9830 & GBIF \\
\hline MX & Chiapas & UMAA & 95434 & 15.3210 & -92.6620 & GBIF \\
\hline$M X$ & Chiapas & USNM & 110522 & 17.5080 & -91.9810 & GBIF \\
\hline MX & Chiapas & $\mathrm{IHN}$ & 1008 / 264710 / U014 & 16.7510 & -93.1150 & GBIF \\
\hline MX & Chiapas & LACM & 9501 & 15.0140 & -92.4010 & GBIF \\
\hline MX & Chiapas & UMAA & 87663 / 285550 / U014 & 15.3790 & -92.4860 & GBIF \\
\hline MX & Chiapas & $\mathrm{IHN}$ & 1847 / 288861 / U014 & 15.8020 & -93.0690 & GBIF \\
\hline
\end{tabular}


Table 1. Continued.

\begin{tabular}{|c|c|c|c|c|c|c|}
\hline Country & State & Collection & Catalog number & Latitude & Longitude & Source \\
\hline$M X$ & Chiapas & $\mathrm{IHN}$ & 28 / 4573 / V050 & 16.7530 & -93.1170 & GBIF \\
\hline$M X$ & Chiapas & USNM & 110522.6082442 & 17.5424 & -91.9916 & GBIF \\
\hline$M X$ & Chiapas & MVZ & 132938 / 292922 / U014 & 15.0150 & -92.2440 & GBIF \\
\hline MX & Chiapas & LACM & 9502 & 15.3808 & -92.7599 & GBIF \\
\hline$M X$ & Chiapas & LACM & 9502 / 265003 / U014 & 15.4060 & -92.8220 & GBIF \\
\hline$M X$ & Chiapas & CM & 88751 & 16.9600 & -91.7800 & GBIF \\
\hline MX & Chiapas & CAS & 140967 & 16.0308 & -93.7500 & GBIF \\
\hline$M X$ & Chiapas & CAS & 140973 & 17.0674 & -92.8611 & GBIF \\
\hline$M X$ & Chiapas & IUMNH & 6287 / 296626 / U014 & 16.0300 & -92.0100 & GBIF \\
\hline MX & Chiapas & $\mathrm{IHN}$ & 757 / 264526 / U014 & 16.7580 & -93.1100 & GBIF \\
\hline$M X$ & Chiapas & ND & 278125 / U014 & 16.1160 & -91.7300 & GBIF \\
\hline $\mathrm{MX}$ & Chiapas & LSUMZ & 110526 & 17.5170 & -91.9830 & GBIF \\
\hline$M X$ & Chiapas & LSUMZ & 110525 & 17.5170 & -91.9830 & GBIF \\
\hline$M X$ & Chiapas & ND & 2474 / EE005 & 15.8100 & -93.0700 & GBIF \\
\hline$M X$ & Chiapas & $\mathrm{IHN}$ & 658 / 264452 / U014 & 16.7580 & -93.1100 & GBIF \\
\hline$M X$ & Chiapas & $\mathrm{IHN}$ & 1368 / 265084 / U014 & 15.3200 & -92.6610 & GBIF \\
\hline$M X$ & Chiapas & UMAA & 123042 & 16.7270 & -93.1000 & GBIF \\
\hline$M X$ & Chiapas & IUMNH & 83096 & 15.4330 & -92.5940 & GBIF \\
\hline$M X$ & Chiapas & iNaturalist & 738605 & 16.8574 & -93.3325 & GBIF \\
\hline$M X$ & Chiapas & iNaturalist & 2765063 & 17.3391 & -91.5463 & GBIF \\
\hline$M X$ & Chiapas & USNM & 110526.6082446 & 17.5424 & -91.9916 & GBIF \\
\hline$M X$ & Chihuahua & - & - & 27.3943 & -108.5358 & LEMOS-ESPINAL \& SMITH 2009 \\
\hline MX & Colima & LACM & 58914 & 19.1906 & -103.7502 & GBIF \\
\hline$M X$ & Colima & LACM & 2545 & 19.3006 & -103.5102 & GBIF \\
\hline$M X$ & Estado de México & $\mathrm{AMNH}$ & 71361 & 18.8334 & -99.6831 & GBIF \\
\hline$M X$ & Guerrero & MVZ & 45089 & 17.5521 & -99.5009 & GBIF \\
\hline MX & Guerrero & MCZ & $\mathrm{R}-823$ & 16.8607 & -99.8901 & GBIF \\
\hline$M X$ & Guerrero & MCZ & R-167181 & 17.4900 & -99.3300 & GBIF \\
\hline$M X$ & Guerrero & CAS & 154315 & 17.6607 & -101.6002 & GBIF \\
\hline $\mathrm{MX}$ & Guerrero & MCZ & $\mathrm{R}-33652$ & 17.5557 & -99.5034 & GBIF \\
\hline$M X$ & Guerrero & MVZ & 45088 & 17.5521 & -99.5009 & GBIF \\
\hline MX & Guerrero & CAS & 135271 & 16.8507 & -99.4001 & GBIF \\
\hline$M X$ & Guerrero & MCZ & $R-33651$ & 17.5557 & -99.5034 & GBIF \\
\hline$M X$ & Hidalgo & - & - & 21.1258 & -98.4110 & RAMÍREZ-BAUTISTA et al. 2014 \\
\hline MX & Jalisco & LACM & 2544 & 19.4006 & -103.3902 & GBIF \\
\hline MX & Jalisco & LACM & 136823 & 19.3606 & -104.9002 & GBIF \\
\hline$M X$ & Jalisco & CAS & 132196 & 20.6605 & -105.2403 & GBIF \\
\hline MX & Jalisco & TCWC & 48057 & 19.8734 & -104.3066 & GBIF \\
\hline MX & Michoacán & MZFC & 12720 & 19.1640 & -101.8700 & GBIF \\
\hline$M X$ & Michoacán & MZFC & 12741 & 19.1640 & -101.8700 & GBIF \\
\hline MX & Morelos & ENCB & 12282 & 19.0130 & -99.2930 & GBIF \\
\hline$M X$ & Morelos & ENCB & 7336 & 18.9680 & -99.1840 & GBIF \\
\hline$M X$ & Nayarit & USNM & 237779.6091644 & 21.2700 & -106.2300 & GBIF \\
\hline$M X$ & Nayarit & - & - & 21.2368 & -104.8996 & ZWEIFEL 1959 \\
\hline$M X$ & Nayarit & LACM & 8733 & 21.6205 & -105.1603 & GBIF \\
\hline MX & Nayarit & CAS & 23824 & 21.6405 & -105.1403 & GBIF \\
\hline MX & Nayarit & CAS & 95756 & 21.6005 & -105.1903 & GBIF \\
\hline$M X$ & Nayarit & SDNHM & 48139 & 21.6700 & -105.1100 & GBIF \\
\hline MX & Nayarit & SDNHM & 56949 & 21.5500 & -105.2400 & GBIF \\
\hline$M X$ & Nayarit & CAS & 23825 & 21.6005 & -105.2003 & GBIF \\
\hline MX & Nayarit & CAS & 23826 & 21.6005 & -105.2003 & GBIF \\
\hline MX & Nayarit & LACM & 103519 & 21.5505 & -105.2403 & GBIF \\
\hline$M X$ & Nayarit & LACM & 125579 & 21.6205 & -105.1603 & GBIF \\
\hline MX & Nayarit & MVZ & 70289 & 21.5399 & -105.2273 & GBIF \\
\hline$M X$ & Nayarit & LACM & 103516 & 22.1405 & -105.2403 & GBIF \\
\hline$M X$ & Nayarit & CAS & 95757 & 21.5505 & -105.2503 & GBIF \\
\hline$M X$ & Nayarit & CAS & 95758 & 21.6505 & -105.1203 & GBIF \\
\hline$M X$ & Nayarit & LACM & 74036 & 21.5705 & -105.2405 & GBIF \\
\hline$M X$ & Nayarit & LACM & 136824 & 21.6905 & -105.0703 & GBIF \\
\hline$M X$ & Nayarit & MVZ & 76710 & 21.6895 & -105.0789 & GBIF \\
\hline$M X$ & Nayarit & LACM & 103515 & 21.8405 & -105.1303 & GBIF \\
\hline
\end{tabular}


Table 1. Continued.

\begin{tabular}{|c|c|c|c|c|c|c|}
\hline Country & State & Collection & Catalog number & Latitude & Longitude & Source \\
\hline$M X$ & Nayarit & LACM & 125578 & 21.6205 & -105.1603 & GBIF \\
\hline$M X$ & Nayarit & LACM & 103517 & 21.6805 & -105.1003 & GBIF \\
\hline$M X$ & Nayarit & MVZ & 71343 & 21.5658 & -105.2375 & GBIF \\
\hline$M X$ & Nayarit & CAS & 259466 & 21.5405 & -105.2883 & GBIF \\
\hline$M X$ & Nayarit & MVZ & 70288 & 21.5546 & -105.2436 & GBIF \\
\hline$M X$ & Nayarit & LACM & 6883 & 22.4605 & -105.3803 & GBIF \\
\hline$M X$ & Nayarit & LACM & 103518 & 21.5805 & -105.2203 & GBIF \\
\hline$M X$ & Nayarit & LACM & 136825 & 21.6505 & -105.1303 & GBIF \\
\hline$M X$ & Nayarit & LACM & 35857 & 21.5505 & -105.2703 & GBIF \\
\hline$M X$ & Nayarit & LACM & 74037 & 21.6005 & -105.1803 & GBIF \\
\hline$M X$ & Oaxaca & MCZ & R-27799 & 16.3708 & -94.1900 & GBIF \\
\hline$M X$ & Oaxaca & UTEP & $\mathrm{H}-8309$ & 16.3908 & -94.1300 & GBIF \\
\hline$M X$ & Oaxaca & LACM & 38187 & 16.4708 & -94.8300 & GBIF \\
\hline$M X$ & Oaxaca & UCM & 39940 & 16.3808 & -96.3400 & GBIF \\
\hline$M X$ & Oaxaca & CAS & 169589 & 16.3708 & -94.1700 & GBIF \\
\hline$M X$ & Oaxaca & UAZ & 27030 & 18.1520 & -97.1010 & GBIF \\
\hline$M X$ & Oaxaca & $\mathrm{KU}$ & 58101 & 16.4308 & -95.0500 & GBIF \\
\hline$M X$ & Oaxaca & LACM & 38186 & 16.4408 & -94.7200 & GBIF \\
\hline$M X$ & Oaxaca & UTEP & $\mathrm{H}-6006$ & 16.4308 & -94.2600 & GBIF \\
\hline$M X$ & Oaxaca & $\mathrm{KU}$ & 58102 & 16.2841 & -95.2333 & GBIF \\
\hline$M X$ & Oaxaca & MVZ & 172391 & 17.0166 & -97.9142 & GBIF \\
\hline$M X$ & Oaxaca & MCZ & $R-27801$ & 16.3708 & -94.1900 & GBIF \\
\hline$M X$ & Oaxaca & LACM & 38188 & 16.4108 & -95.0800 & GBIF \\
\hline$M X$ & Puebla & BUAP & 1839 & 18.1680 & -97.4680 & GBIF \\
\hline$M X$ & Querétaro & MZFC & 7547 & 26.2690 & -99.0500 & GBIF \\
\hline$M X$ & Quintana roo & UMMZ & 113562 & 19.5830 & -88.0330 & GBIF \\
\hline$M X$ & Quintana Roo & KU & 157575 & 19.3407 & -88.0698 & GBIF \\
\hline$M X$ & Sinaloa & LACM & 6860 & 23.4104 & -106.4003 & GBIF \\
\hline$M X$ & Sinaloa & iNaturalist & 395071 & 23.3527 & -106.4750 & GBIF \\
\hline$M X$ & Sinaloa & LACM & 6864 & 23.5104 & -106.6303 & GBIF \\
\hline$M X$ & Sinaloa & MVZ & 71340 & 23.1945 & -106.2091 & GBIF \\
\hline$M X$ & Sinaloa & LACM & 103526 & 24.0904 & -106.8203 & GBIF \\
\hline$M X$ & Sinaloa & CAS & 138867 & 23.9204 & -106.8903 & GBIF \\
\hline$M X$ & Sinaloa & MCZ & R-61419 & 23.4504 & -106.4203 & GBIF \\
\hline$M X$ & Sinaloa & MVZ & 71338 & 23.4661 & -106.4764 & GBIF \\
\hline$M X$ & Sinaloa & MVZ & 71342 & 23.1180 & -106.0388 & GBIF \\
\hline$M X$ & Sinaloa & LACM & 6866 & 24.4104 & -106.7003 & GBIF \\
\hline$M X$ & Sinaloa & $\mathrm{KU}$ & 67673 & 24.1004 & -106.8303 & GBIF \\
\hline$M X$ & Sinaloa & $\mathrm{KU}$ & 73532 & 24.9003 & -107.4003 & GBIF \\
\hline$M X$ & Sinaloa & $\mathrm{KU}$ & 78941 & 23.4504 & -105.8403 & GBIF \\
\hline$M X$ & Sinaloa & LACM & 6872 & 25.5833 & -107.3003 & GBIF \\
\hline$M X$ & Sinaloa & LACM & 6886 & 23.0904 & -106.0103 & GBIF \\
\hline$M X$ & Sinaloa & CAS & 23822 & 23.9404 & -106.8703 & GBIF \\
\hline$M X$ & Sinaloa & LACM & 6862 & 23.4604 & -106.4603 & GBIF \\
\hline$M X$ & Sinaloa & LACM & 6876 & 23.9904 & -106.9703 & GBIF \\
\hline$M X$ & Sinaloa & $\mathrm{KU}$ & 73530 & 23.3104 & -106.4003 & GBIF \\
\hline$M X$ & Sinaloa & CAS & 23823 & 22.8004 & -105.7103 & GBIF \\
\hline$M X$ & Sinaloa & LACM & 6877 & 23.3604 & -106.1303 & GBIF \\
\hline$M X$ & Sinaloa & LACM & 103520 & 23.2504 & -106.4203 & GBIF \\
\hline$M X$ & Sinaloa & LACM & 103528 & 22.5205 & -105.4303 & GBIF \\
\hline$M X$ & Sinaloa & LACM & 136826 & 23.3204 & -106.0003 & GBIF \\
\hline$M X$ & Sinaloa & LACM & 6874 & 24.7004 & -107.3603 & GBIF \\
\hline$M X$ & Sinaloa & LACM & 125321 & 23.5604 & -106.4703 & GBIF \\
\hline$M X$ & Sinaloa & SBMNH & 208 & 23.1931 & -106.2492 & GBIF \\
\hline$M X$ & Sinaloa & LACM & 6880 & 23.3304 & -106.4103 & GBIF \\
\hline$M X$ & Sinaloa & $\mathrm{KU}$ & 174805 & 23.9204 & -106.8903 & GBIF \\
\hline$M X$ & Sinaloa & LACM & 103521 & 23.1604 & -106.2203 & GBIF \\
\hline$M X$ & Sinaloa & CAS & 23821 & 24.2204 & -107.0503 & GBIF \\
\hline$M X$ & Sinaloa & MVZ & 70286 & 23.4417 & -106.4647 & GBIF \\
\hline$M X$ & Sinaloa & MCZ & $\mathrm{R}-61421$ & 23.5104 & -106.3903 & GBIF \\
\hline$M X$ & Sinaloa & $\mathrm{KU}$ & 80752 & 23.7604 & -106.6503 & GBIF \\
\hline
\end{tabular}


Table 1. Continued.

\begin{tabular}{|c|c|c|c|c|c|c|}
\hline Country & State & Collection & Catalog number & Latitude & Longitude & Source \\
\hline$M X$ & Sinaloa & LACM & 6863 & 23.6304 & -106.5803 & GBIF \\
\hline$M X$ & Sinaloa & LACM & 6871 & 24.4404 & -106.7003 & GBIF \\
\hline$M X$ & Sinaloa & CAS & 159373 & 24.7651 & -107.3617 & GBIF \\
\hline$M X$ & Sinaloa & MVZ & 71339 & 23.4998 & -106.4900 & GBIF \\
\hline$M X$ & Sinaloa & KU & 73531 & 24.1904 & -107.1103 & GBIF \\
\hline$M X$ & Sinaloa & LACM & 6885 & 23.0904 & -106.0103 & GBIF \\
\hline$M X$ & Sinaloa & LACM & 6875 & 24.2704 & -107.2803 & GBIF \\
\hline$M X$ & Sinaloa & LACM & 6884 & 23.0904 & -106.0103 & GBIF \\
\hline$M X$ & Sinaloa & LACM & 126260 & 23.5204 & -106.4903 & GBIF \\
\hline$M X$ & Sinaloa & MVZ & 70287 & 23.6765 & -106.5896 & GBIF \\
\hline$M X$ & Sinaloa & LACM & 6865 & 23.4804 & -106.3903 & GBIF \\
\hline$M X$ & Sinaloa & LACM & 25912 & 23.2904 & -106.2203 & GBIF \\
\hline$M X$ & Sinaloa & KU & 73528 & 23.3304 & -106.4203 & GBIF \\
\hline$M X$ & Sinaloa & MCZ & R-61418 & 23.4104 & -106.3403 & GBIF \\
\hline$M X$ & Sinaloa & CAS & 159374 & 23.6504 & -106.6903 & GBIF \\
\hline$M X$ & Sinaloa & CAS & 95759 & 24.7004 & -107.3603 & GBIF \\
\hline$M X$ & Sinaloa & LACM & 6868 & 25.4703 & -107.3803 & GBIF \\
\hline$M X$ & Sinaloa & LACM & 6887 & 23.0904 & -106.0103 & GBIF \\
\hline$M X$ & Sinaloa & LACM & 76590 & 23.2604 & -106.1203 & GBIF \\
\hline$M X$ & Sinaloa & LACM & 103522 & 24.1904 & -107.2203 & GBIF \\
\hline$M X$ & Sinaloa & LACM & 103525 & 23.1604 & -105.8703 & GBIF \\
\hline$M X$ & Sinaloa & UTEP & $\mathrm{H}-5174$ & 23.1904 & -106.2703 & GBIF \\
\hline$M X$ & Sinaloa & MVZ & 71341 & 23.1799 & -106.2202 & GBIF \\
\hline$M X$ & Sinaloa & LACM & 6858 & 23.4104 & -106.4503 & GBIF \\
\hline$M X$ & Sinaloa & LACM & 103523 & 24.8004 & -107.3903 & GBIF \\
\hline$M X$ & Sinaloa & LACM & 103524 & 24.8004 & -107.3903 & GBIF \\
\hline$M X$ & Sinaloa & KU & 73529 & 23.5604 & -106.5103 & GBIF \\
\hline$M X$ & Sinaloa & LACM & 6859 & 23.4704 & -106.3903 & GBIF \\
\hline$M X$ & Sinaloa & LACM & 6867 & 25.4303 & -107.4603 & GBIF \\
\hline$M X$ & Sinaloa & LACM & 7244 & 23.0904 & -106.0103 & GBIF \\
\hline$M X$ & Sinaloa & LACM & 103527 & 22.5405 & -105.5103 & GBIF \\
\hline$M X$ & Sinaloa & LACM & 6869 & 23.4604 & -106.3203 & GBIF \\
\hline$M X$ & Sinaloa & LACM & 6873 & 23.0404 & -105.8503 & GBIF \\
\hline$M X$ & Sinaloa & CAS & 23820 & 25.1907 & -107.8462 & GBIF \\
\hline$M X$ & Sinaloa & MCZ & $\mathrm{R}-61420$ & 23.5104 & -106.3903 & GBIF \\
\hline$M X$ & Sinaloa & LACM & 6870 & 24.1204 & -106.7503 & GBIF \\
\hline$M X$ & Sinaloa & LACM & 6882 & 25.7303 & -107.2503 & GBIF \\
\hline$M X$ & Sinaloa & LACM & 103529 & 22.5105 & -105.4103 & GBIF \\
\hline$M X$ & Sinaloa & LACM & 6878 & 22.5338 & -105.7336 & GBIF \\
\hline$M X$ & Sinaloa & LACM & 121326 & 25.1803 & -107.9803 & GBIF \\
\hline$M X$ & Sinaloa & CAS & 105002 & 24.8212 & -107.4942 & GBIF \\
\hline$M X$ & Sinaloa & KU & 68752 & 26.8403 & -108.2604 & GBIF \\
\hline$M X$ & Sinaloa & LACM & 6861 & 24.0904 & -107.0403 & GBIF \\
\hline$M X$ & Sinaloa & LACM & 6879 & 23.4504 & -106.4603 & GBIF \\
\hline$M X$ & Sinaloa & LACM & 6881 & 24.1204 & -107.0703 & GBIF \\
\hline$M X$ & Sonora & MVZ & 137741 & 27.0667 & -109.0414 & GBIF \\
\hline$M X$ & Sonora & CAS & 138868 & 27.0203 & -108.9304 & GBIF \\
\hline$M X$ & Sonora & LACM & 103530 & 27.0811 & -109.0815 & GBIF \\
\hline$M X$ & Sonora & UAZ & UAZ 56042-PSV & 28.4079 & -109.1125 & GBIF \\
\hline$M X$ & Tamaulipas & MNHUK & 174806 & 22.8920 & -98.0730 & GBIF \\
\hline$M X$ & Veracruz & MCZ & R-45679 & 18.8840 & -96.7834 & GBIF \\
\hline$M X$ & Veracruz & CUMV & 12631 & 18.5340 & -95.6500 & GBIF \\
\hline$M X$ & Veracruz & UCM & 48112 & 21.8305 & -97.7601 & GBIF \\
\hline$M X$ & Veracruz & CAS & 132163 & 20.2073 & -96.7767 & GBIF \\
\hline$M X$ & Veracruz & $\mathrm{KU}$ & 140062 & 19.5006 & -96.8356 & GBIF \\
\hline$M X$ & Veracruz & UTEP & $\mathrm{H}-6005$ & 18.4840 & -95.0667 & GBIF \\
\hline$M X$ & Yucatán & MCZ & $\mathrm{R}-7245$ & 21.2839 & -89.6666 & GBIF \\
\hline$M X$ & Yucatán & $\mathrm{CM}$ & 49652 & 20.6906 & -88.5799 & GBIF \\
\hline$M X$ & Yucatán & $\mathrm{CM}$ & 46930 & 20.6906 & -88.5799 & GBIF \\
\hline$M X$ & Yucatán & MCZ & R-7239 & 20.6673 & -88.5665 & GBIF \\
\hline$M X$ & Yucatán & FMNH & 36243 & 20.6830 & -88.6830 & GBIF \\
\hline
\end{tabular}


Bañuelos-Alamillo et al. | Imantodes gemmistratus: new state record for Zacatecas, Mexico

Table 1. Continued.

\begin{tabular}{|c|c|c|c|c|c|c|}
\hline Country & State & Collection & Catalog number & Latitude & Longitude & Source \\
\hline$M X$ & Yucatán & UMMZ & 80936 & 20.6830 & -88.5670 & GBIF \\
\hline$M X$ & Yucatán & FMNH & 36239 & 20.7000 & -88.8170 & GBIF \\
\hline$M X$ & Yucatán & $\mathrm{KU}$ & 157576 & 20.4106 & -89.7799 & GBIF \\
\hline$M X$ & Yucatán & FMNH & 153577 & 21.0970 & -89.5990 & GBIF \\
\hline$M X$ & Yucatán & FMNH & 20602 & 20.6830 & -88.5670 & GBIF \\
\hline$M X$ & Yucatán & FMNH & 20601 & 20.6830 & -88.5670 & GBIF \\
\hline$M X$ & Yucatán & UMMZ & 73033 & 20.6830 & -88.5670 & GBIF \\
\hline$M X$ & Yucatán & FMNH & 153577 & 21.0330 & -89.5670 & GBIF \\
\hline$M X$ & Yucatán & FMNH & 36242 & 20.7000 & -88.8170 & GBIF \\
\hline$M X$ & Yucatán & FMNH & 36240 & 20.7000 & -88.8170 & GBIF \\
\hline$M X$ & Yucatán & ND & NA / 2272 / W030 & 20.9670 & -89.9170 & GBIF \\
\hline$M X$ & Yucatán & $\mathrm{CMNH}$ & 46930 & 20.7000 & -88.7500 & GBIF \\
\hline$M X$ & Yucatán & FMNH & 36241 & 20.6830 & -88.6830 & GBIF \\
\hline$M X$ & Yucatán & $\mathrm{KU}$ & 171699 & 20.9406 & -88.6799 & GBIF \\
\hline$M X$ & Zacatecas & SDSNH_HerpPC & 05340 & 22.6847 & -103.7345 & This study \\
\hline PA & Chame & AMNH & 90020 & 8.5555 & -79.8688 & GBIF \\
\hline SV & San Salvador & $\mathrm{KU}$ & 183890 & 13.6667 & -89.1000 & GBIF \\
\hline SV & San Salvador & $\mathrm{KU}$ & 62100 & 13.7000 & -89.2000 & GBIF \\
\hline SV & San Salvador & $\mathrm{KU}$ & 183889 & 13.6667 & -89.1000 & GBIF \\
\hline SV & San Salvador & $\mathrm{KU}$ & 62099 & 13.7000 & -89.2000 & GBIF \\
\hline
\end{tabular}

